\title{
Resignificación socioespacial y construcción de subjetividad. Personas sin hogar en la Ciudad de Buenos Aires
}

\author{
Sociospatial re-signification and the \\ construction of subjectivity. Homeless \\ persons in the City of Buenos Aires"
}

Griselda Palleres ${ }^{1}$

Aceptación: 20 septiembre 2009

Aprobación: 20 mayo 2010

\section{RESUMEN}

El vivir en la calle utilizando los espacios públicos ya no es un problema que comprenda a adultos solos. Es creciente la posibilidad de encontrar niños, jóvenes y hasta familias completas que se encuentran en la denominada "situación de calle". Si bien se trata de un problema en franco aumento y multidimensional, y pese a que es considerada la forma más extrema y más visible de exclusión social, las conceptualizaciones que se producen alrededor de las personas sin hogar no tienen en cuenta los aspectos sustanciales de la realidad que a diario enfrentan sus intervinientes. De esta manera, el movimiento a lo largo de la ciudad por parte de los sujetos se carga de atributos negativos y de cualidades exteriores que los señalan como peligrosos y culpables de su situación. Este artículo propone dar cuenta del modo en que las personas sin hogar, a través del desarrollo de diversas tácticas, transforman y cargan de significación su transcurrir diario, el espacio público en el que desarrollan su cotidianeidad y su subjetividad.

Palabras clave: Personas sin hogar, espacio público, subjetividad.

\begin{abstract}
Living in the street using public spaces is not a problem involving adults alone. The possibility of finding children, young people and even whole families living in the so-called "situation of street" is increasing. While this is a growing problem and multidimensional, and while is considered as a more extreme and more visible social, the conceptualizations that occur around homeless people do not take into account the substantial aspects of the reality daily faced by its speakers. Thus, the movement of these subjects around the city is loaded of negative attributes and exterior qualities that point them as dangerous and guilty of their situation. This article proposes realizing how the homeless, through the development of various tactics, transform and load of significance their daily passing, the public space in which they carry out their daily life and subjectivity.
\end{abstract}

Key words: Homeless people, public space, subjectivity.

Licenciada en Ciencias Antropológicas de la Universidad de Buenos Aires y Especialista en Gestión de Proyectos Sociales de la Universidad Nacional de Cuyo, Becaria del Consejo Nacional de Investigaciones Científicas y Técnicas (CONICET) y docente de la Facultad de Ciencias Sociales (UBA). griselda.palleres@gmail.com 


\section{Introducción}

En las últimas décadas las ciudades han experimentado masivos y paralelos cambios en la economía nacional, en la estructura social y en la organización espacial. A comienzos de la década de $1990^{2}$ el Estado argentino redefinió su rol en un nuevo modelo de crecimiento y de desarrollo que se caracterizó por la apertura económica y su reorientación hacia el mercado externo, por una modernización tecnológica importante y por políticas de ajuste que terminaron produciendo el aumento del desempleo y la reducción del gasto público en coberturas sociales (Svampa 2005). Estas transformaciones desencadenaron distintos procesos de desigualdad y de exclusión social que no solo afectaron a un número creciente de la población, sino que también produjeron importantes mutaciones en la estructura social a través de la generación de nuevas formas de jerarquización social y de segregación espacial (Althabe 2005, Roberts 2007).

Por un lado, el aumento de la exclusión social se manifiesta en una progresiva precariedad de las estructuras sociales que afecta a todos los aspectos de la vida, tanto el acceso a los medios materiales necesarios para la subsistencia (vivienda, escuelas, hospitales, transportes), como las formas mismas de convivencia y los mecanismos de gestión social y cultural. Por su parte, los procesos de segregación socioespacial han disminuido los espacios de interacción entre sectores de orígenes sociales distintos, produciendo una espacialización de las relaciones sociales con la constitución de fronteras sociales cada vez más rígidas (Roberts 2007, Svampa 2005). Por añadidura, se produce un debilitamiento de los espacios públicos que expresan un cambio significativo de las representaciones sociales. La limitación a ciertas dimensiones de la sociabilidad instaura cada vez más espacios urbanos y se atribuye al inmigrante y al trabajador ilegal, a las personas sin hogar, al villero, a los jóvenes, a los ancianos, a las personas encarceladas, etc. Se trata de una situación de aislamiento que niega a los habitantes de estos conjuntos la posibilidad de interacción y de reconocimiento mutuo, es decir, el acceso a uno de los principios fundamentales la vida urbana y de los derechos ciudadanos. La consecuencia es hoy ciudades sin ciudadanos - los sin derecho y ciudadanos sin ciudad- los autoexcluidos por miedo en barrios cerrados, protegidos por rejas, púas, guardias, cámaras y barreras controladas que impiden el acceso y el libre tránsito. Se trastoca así el sentido mismo de la ciudad como espacio de convivencia humana, de encuentro, de intercambio, de complementación y de enriquecimiento intercultural (Hidalgo Dattwyler 2007, Velásquez 2007).

En este marco, la permanencia de las personas sin hogar en los espacios públicos se debate entre la conformación de estereotipos, de prejuicios y de situaciones de exclusión, respondiendo a varias imágenes culturales que las posiciona en la "condición humana más marginal" ya que existe la creencia de que no comparten la cultura dominante.

Si se tiene en cuenta que construir la cotidianeidad viviendo en las calles implica la obligación de realizar en los espacios públicos actividades reservadas para el ámbito privado, entonces de comprenderá que la delimitación entre la esfera pública y la privada se presenta tan difusa como contradictoria. Frente a esto, la persona sin hogar ha sido representada como la figura límite con relación a lo público $\mathrm{y}$, su aspecto exterior, marca la distinción entre la sociedad armónica y la sociedad de los excluidos, conformándose como una totalidad o identidad social (Kawash 1998). Como resultado de esto último, los sujetos son definidos no solo por sus propiedades espaciales, sino también por sus particularidades materiales y corporales. De igual forma, para el la sociedad, el cuerpo de las personas sin hogar y su continuo movimiento se presenta como una amenaza constante para el espacio público y el uso del

Si bien fue a partir de abril de 1991 cuando en la Argentina se implementó una política económica fundada en el pensamiento neoclásico, en términos ideológicos, esta continuaba con las líneas implementadas en 1976. En términos de Svampa, "los cambios en el orden económico arrancan durante la década del 70, a partir de la instalación de regímenes militares en el Cono Sur de América Latina; las transformaciones operadas en la estructura social comenzarán a tornarse visibles en la década del 80 , durante los primeros años de retorno a la democracia; por último, podemos situar los cambios mayores a fines de los 80 y principios de los 90, con la gestión menemista” (Svampa, 2005: 22). 
mismo (Kawash 1998). Dicha amenaza es a la vez simbólica - con su sola presencia la persona cambia la definición de los espacios-y física porque el cuerpo utiliza los espacios. Se produce así una doble minimización entre lo público y lo corporal: la persona se ve minimizada en su ser y en su extensión.

Así es como el continuo transitar a lo largo de la ciudad, utilizando los espacios públicos, se carga de atributos negativos y de cualidades exteriores que produce y precipita el surgimiento de: 1) conceptualizaciones que responden a una gran cantidad de prejuicios e imaginarios conformados a través de la memoria colectiva y del sentido común, y 2) categorizaciones que señalan al individuo como enfermo, peligroso y culpable de su situación (Palleres 2004).

Cabe destacar que, en la actualidad, muchas de estas conceptualizaciones surgen de la utilización inapropiada de instrumentos de evaluación psiquiátrica ${ }^{3}$. Los resultados de estos exámenes, en su gran mayoría, aluden a un individuo con una "sociabilidad defectuosa" y que padece desordenes mentales (Snow y Anderson 1993, Snow y Mulcahy 2001, O'Sullivan 2008). Como consecuencia, se obtiene una imagen descontextualizada e incompleta de la problemática y que se relaciona más con imaginarios sociales que con las circunstancias que las personas sin hogar deben enfrentar cotidianamente.

Este tipo de explicaciones no tienen en cuenta los aspectos sustanciales de la realidad que a diario enfrentan sus intervinientes $\mathrm{ni}$ tampoco consideran el espacio vital donde se desarrolla la sociabilidad y las interacciones de los sujetos. Por estos motivos el espacio público termina siendo concebido como aquel lugar donde las personas que viven en la calle solo tienen la posibilidad de sobrevivir. Bajo esta perspectiva el sujeto se encuentra totalmente individualizado y expuesto por la falta de vínculos y de sostenes relacionados con el trabajo, la transmisión familiar, la posibilidad de construirse un futuro. En otros términos, las personas sin hogar resultan política, social e individualmente ubicadas en la periferia de la sociedad.

Este artículo no se propone presentar una visión límite sobre la construcción de la cotidianeidad de las personas sin hogar, sino que, por el contrario, procura dar cuenta de la elaboración y la puesta en práctica de diversas tácticas por parte de los sujetos, que les brindan la posibilidad de transformar tanto el espacio urbano como los significados sobre sí mismos.

\section{Sin hogar en la ciudad y vida cotidiana}

En esta sección, el enfoque etnográfico permite dar cuenta de las prácticas cotidianas y del modo en que los sujetos insertos en diversas dinámicas socioespaciales continuamente manipulan, transforman y cargan de significación no solo su entorno urbano, sino también su propia subjetividad.

Es apropiado señalar que no es posible dar cuenta de una única causa o hecho que determine la pérdida del hogar, sino que esta problemática forma parte de un proceso de raíz multicausal y/o multidimensional y que pocas veces sucede de forma abrupta o repentina. Por estas razones los sujetos, en una primera instancia y antes de pernoctar de forma continua en el espacio público, intentan conservar los lazos mantenidos hasta el momento, permaneciendo por un tiempo entre casas de familiares y/o de amigos. No obstante, si la situación no se soluciona con relativa rapidez, comienzan a permanecer más tiempo en los espacios públicos debido a que los vínculos se tornan más endebles $y$, en algunos casos, insostenibles. A partir de ese momento, lo que en un principio se presenta como ocasional o circunstancial, comienza a ser una rutina y entonces se ha dado lo que genéricamente se denomina vivir en la calle.

Los estudios llevados a cabo por los Programas dependientes del Gobierno de la Ciudad de Buenos Aires (GCBA) generalmente se circunscriben a cuestionar la manera en que la persona llega a conformarse como sin techo y los "grados de conciencia" respecto a su situación precaria. De esta forma, para las instituciones, el vivir en la calle se relaciona directamente con la inestabilidad psíquica de los individuos y que, en muchos casos, esto los conduce a la bebida y a la droga. Una muestra clara de esto lo constituyen los siguientes trabajos: CALCAGNO, L. (1999). Los que duermen en la calle. Un abordaje de la indigencia extrema en la ciudad de Buenos Aires, y MALANCA, P. (coord.) (2003). Personas sin techo. Algunas consideraciones psicológicas preliminares en el abordaje de trabajo de calle. Ambas publicaciones pertenecen al Centro de Documentación en Políticas Sociales del Gobierno de la Ciudad de Buenos Aires. 


\section{Transcurrir diario y espacio público}

Desde el primer momento en que se produce la pérdida del hogar, se genera una fisura entre lo que ellos consideran su vida normal y la actual situación que se caracteriza por tener que vivir en la calle cotidianamente. Si bien, como se señaló anteriormente, este proceso no se produce abruptamente, este les exige responder a situaciones que nunca imaginaron, ya que las habilidades adquiridas con anterioridad no responden favorablemente a las nuevas circunstancias.

Existe la creencia de que la cotidianeidad de las personas sin hogar se caracteriza por la inactividad, por el desorden y por situaciones de tránsito sin un objetivo aparente. Sin embargo, el estar viviendo en la calle es la parte visible de todo un andar, un movimiento en busca de recursos "negados" y que responde a varios objetivos que van desde la satisfacción de necesidades inmediatas para subsistir, hasta otras más mediatas como la búsqueda de un rincón de la ciudad del cual poder adueñarse o sentir como propio a lo largo del tiempo. El hecho de que la movilidad a través del espacio público la realicen en tiempos que no se encuentran reglamentados por el trabajo u otro tipo de obligaciones sociales, no significa que carezcan de una rutina fiable con respecto a lo que hacen a lo largo del día.

Por el contrario, cotidianamente, las personas sin hogar viven insertas en un movimiento que remite a un círculo o circuito. Se trata de un proceso dinámico que atraviesa etapas a lo largo de la jornada y que depende de la conservación de un trabajo informal o bien de la inclusión en las redes instituciona- les. Esto último se debe a que cuando ya no existen los compromisos laborales ${ }^{4}-0$ estos son escasos-, casi toda la jornada es atravesada por los distintos servicios que brindan las instituciones ya sea de dependencia gubernamental, religiosa ${ }^{5}$ o de la sociedad civil. En el ámbito de la Ciudad de Buenos Aires la red de alojamiento nocturno depende principalmente del Gobierno de la Ciudad de Buenos Aires (GCBA) a través de los hogares de tránsito y de los paradores nocturnos ${ }^{6}$. En estos establecimientos las personas sin hogar tienen la posibilidad de pernoctar por una noche o por un periodo limitado de tiempo. Por otra parte, la obtención de vestimenta y la posibilidad de bañarse, generalmente, la realizan a través de las redes que conforman las organizaciones no gubernamentales. Entonces, si las personas sin hogar en algunos lugares tienen la posibilidad de dormir, en otros la de comer y en otros diferentes la de bañarse, es posible decir que estas redes institucionales convierten al espacio en una especie de casa extendida a lo largo de la ciudad. En consecuencia, el acceso a la ayuda que brindan las instituciones y la modalidad de trabajo que poseen marcan aún más la amplitud del espacio ya que lleva a las personas a recorrer largos trayectos a fin de satisfacer sus necesidades inmediatas. Para acceder a cada uno de estos servicios deben estar en continuo movimiento, perpetuando no solo a este último, sino también las rutinas y circuitos cotidianos que fueron conformando a través de su experiencia de vida en la calle.

Paulatinamente deben tomar decisiones cada vez más pragmáticas y funcionales que les permitan responder a las condiciones actuales. Al principio, las personas sin hogar cargan objetos que les traen recuerdos, de-

Es apropiado señalar que para las personas sin hogar, la posibilidad de continuar con ciertas labores les proporciona no solo el acceso a los recursos materiales, sino que también les ayuda a preservar su autoestima. Puede que la ausencia de un trabajo formal las haya llevado a encontrarse en la calle o bien a que la situación resulte de difícil solución. A pesar de que la falta de trabajo no siempre es la causa desencadenante de este estado, en su mayoría argumentan que si tuvieran un trabajo regular y formal podrían aspirar a mejorar su condición. No cabe duda que la ausencia de este sumerge a los sujetos en situaciones inestables que dificultan aún más la inserción laboral. Así, con el transcurrir del tiempo, la situación se carga de mayor incertidumbre y cada vez es más difícil conseguir trabajo. Todo forma parte en mayor o menor medida de un mismo proceso.

5 La mayoría de las personas sin hogar, en mayor o menor medida, mantienen lazos con las redes institucionales. Hay quienes viven cerca de la parroquia donde obtienen alimentos, ropa y ayuda social o permanecen gran parte del día cerca del parador de tránsito donde duermen cada noche; y hay otros que, si bien no recurren a los servicios directamente, reciben algún tipo de asistencia. Es casi imposible encontrar una jornada en que las personas sin hogar no se hayan visto atravesadas en algún aspecto por lo institucional.

6 Es pertinente aclarar que el Gobierno de la Ciudad de Buenos Aires también tiene hogares y paradores convenidos con organizaciones de la sociedad civil. Ejemplo de esto último es el Hogar Monteagudo que es administrado por el Servicio Interparroquial de Ayuda Mutua - SIPAM (Palleres 2009). 
jando los más prescindibles en casa de amigos, de familiares o bien en el mismo andar. Gradualmente, lo que era imprescindible en un momento se va transformando en algo total y completamente prescindible con el correr del tiempo. La necesidad y la obligación de cargar con cada una de sus pertenencias los lleva a desarrollar distintas tácticas que les permita continuar a diario y trasladarse de un lugar a otro. Además, a pesar de cargar con varios bolsos, muchos de ellos suelen vestir gran cantidad de ropa, independientemente de las condiciones climáticas. Esto último se debe a que resulta más cómodo soportar el calor que tener que cargar con un bolso muy pesado. Estas tácticas también se relacionan con el modo de afrontar todos los días el estar viviendo en la calle protegiéndose del frío, de la lluvia o de las inclemencias del tiempo en general. Hay quienes duermen expuestos y solo utilizan cartones para aislar su cuerpo del piso frío, mientras que otros lo hacen dentro de una estructura de cartón ${ }^{7}$. Cada uno sabe muy bien cuál es la mejor manera de aprovechar los beneficios estructurales que les brinda el rincón donde habitualmente duermen. Pero es necesario aclarar que si bien las tácticas les permitan superar la materialidad de la nueva situación, estas no garantizan la satisfacción de la dificultad de vivir en la calle a largo plazo, sino que solo lo hacen en plazos y tiempos inmediatos.

Cuando llevan un cierto tiempo sin hogar no cuentan con un trabajo formal, y a diario, progresivamente, comienzan a disponer de todo el tiempo y de todos los lugares, ya han perdido algunos de los lazos afectivos, y por otra parte, los objetos y hasta el aspecto personal comienzan a deteriorarse. En gran medida, las mismas actividades que les permiten subsistir a diario son la marca exterior que los pone en evidencia de manera visible y que determinará la principal diferencia entre su "situación en calle" y el resto de la sociedad. Es decir, la vestimenta, el acarreo de bolsos o pertenencias, trasladarse de un lugar a otro, la provisión de materiales y la construcción de estructuras espontáneas, las distintas actividades informales que pueda realizar tales como la búsqueda de objetos entre la basura o, en última instancia, el mendigar, señalan a las personas sin hogar como "marginales". Por estos motivos, el estar aquí y ahora, con sus particularidades materiales y corporales es lo que perpetúa el estigma y lo que los define como personas de/en la calle.

No obstante, el hecho de que se forme una imagen estereotipada alrededor de ellos, que alterna entre la búsqueda de la libertad, la locura y la miseria ${ }^{8}$, no significa que quienes se encuentran en esa situación conciban su cotidianeidad de tal forma. Entonces será necesario dar cuenta del modo en que los propios sujetos construyen una imagen sobre sí mismos, superando aquellas conceptualizaciones que remiten a representaciones e imaginarios sociales.

\section{Construcción de sí mismo y búsqueda de la intimidad}

Así como no es posible dar cuenta de una única causa que origine el problema, tampoco lo es hablar de una identidad común y compartida por las personas sin hogar. El problema de la falta de vivienda es un fenómeno que encierra múltiples dimensiones no solo por las causas que lo originan, sino también

En trabajos anteriores se ha observado que las construcciones improvisadas de cartón en las cuales duermen tienen que ver con la necesidad de resguardarse de los transeúntes. Si bien en invierno cuando se registran las temperaturas más bajas utilizan el cartón para protegerse del frío, muchas veces estas especies de fortalezas solo responden a la necesidad de ocultarse y de ser invisibles para el resto de la sociedad. Las estructuras más complejas arquitectónicamente por lo general son realizadas por las mujeres, quienes continuamente intentan obtener mayor privacidad al momento de cambiarse de ropa. Las mujeres tienden a hacer construcciones con los cartones que parecen pequeñas fortalezas en las cuales se ocultan. Por lo tanto, es frecuente que tiendan a apilar cajas enteras de cartón y a dormir dentro de ellas independientemente de que sea invierno o verano. Esto marca, por un lado, la necesidad de privacidad y, por otro, se relaciona con sentimientos de vergüenza y de temor a estar todo el tiempo expuestos a los peligros que representa vivir en la calle (Palleres 2004).

8 A lo largo de la historia, la necesidad de autoexpresión y la búsqueda de una verdad a ser encontrada más allá del trabajo fijo o de la rutina social fueron algunas de las razones que justificaron la figura de la persona que abandona la familia, la vivienda, el trabajo y la vida sedentaria para nomadizarse por cuenta propia. En nuestro país tal fue el caso de los linyeras o los crotos así denominados a partir de 1920- y a quienes se les permitió a través de reglamentaciones gubernamentales viajar gratuitamente en los trenes en busca de trabajo. Si bien puede que muchas personas se hayan dedicado a andar por la vía de la vida en busca de un ideal, también es muy probable que hayan sido obligados a recorrer caminos alternativos en busca de su subsistencia. Sin embargo, a estos siempre se los asoció con rasgos personales socialmente condenados, de modo que fueron vistos como vagos, sucios, delincuentes, alcohólicos o locos (Palleres 2004). 
por la heterogeneidad de quienes lo conforman. Por estas razones, ellos no buscan una inscripción común a la "situación en calle", sino que, contrariamente, ellos intentan borrar esa "etiqueta" continuamente.

Una vez que la cotidianeidad de las personas sin hogar se desarrolla enteramente en las calles de la ciudad, estas deben responder a múltiples variables no solo para subsistir, sino también en tanto personas subjetivas con capacidad de sentir temor, angustia, dolor, felicidad, vergüenza, impotencia, goce, etc. La recurrencia a ciertos lugares elegidos no solo permite el acceso a las necesidades materiales, sino que también es funcional para el establecimiento y conservación de relaciones interpersonales, que se mantienen o inician aun teniendo una vida en la calle. Es marcada la necesidad que ellos sienten de mantener ciertos lazos afectivos que se asemejen a la pertenencia que alguna vez tuvieron en el seno familiar. Estos lazos les recuerdan que, a pesar de no contar con un hogar ni pertenencias, también son personas con sentimientos, con opinión, con formas de pensar y de sentir.

No obstante, la continua exposición lleva a los sujetos a cuestionarse respecto de la falta de pertenencias y lo indigno de no tener la posibilidad de contar con un momento de privacidad. Luchan contra la estigmatización aunque a veces no lo logran ya que, a pesar de la elaboración de distintas tácticas, ellos continúan realizando en los espacios públicos actividades reservadas para el ámbito privado, es decir, siguen construyendo su cotidianeidad en la vía pública.

En trabajos anteriores se ha mostrado que el deseo de higiene personal es una señal de que el sujeto aún no ha llegado al abandono total de sí misma (Palleres 2004). Las personas sin hogar tienen la posibilidad de asearse a diario accediendo a baños públicos en casas de comida rápida, en confiterías o en restaurantes. Pero la higienización que realizan de sus cuerpos no es completa y las marcas que los señalan como "marginales" continúan encarnándose en sus personas. Pese a esto, ellos realizan grandes esfuerzos para eliminar la suciedad y los olores: intentan mantener su vestimenta y sus pertenencias en buenas condiciones, cuidan su aspecto corporal y se mantienen en un movimiento constante para no ser identificados. Estas acciones se repiten diariamente, salvo por un día a la semana que es cuando acceden al servicio de duchas que les brindan las redes institucionales ${ }^{9}$. Quienes acceden a este servicio cuentan con solo quince minutos, aproximadamente ${ }^{10}$ para ducharse debido a que el servicio permanece abierto por un tiempo limitado. Pese a que este acto se da en un tiempo que no es lo suficientemente prolongado y que en un espacio al cual no pueden concurrir tantas veces como deseen, paradójicamente, es en ese momento cuando los sujetos obtienen el reconocimiento y el acceso a la intimidad que no logran en la vida cotidiana. La principal diferencia radica en que las duchas poseen habitáculos individuales y pueden ducharse a puerta cerrada, permitiéndoles confrontarse con su cuerpo completamente desnudo. A pesar de que quince minutos es un tiempo muy escaso para el reconocimiento individual y corporal, se produce un antes y un después de la situación y de la concepción de ellos sobre sí mismos una vez que salen de las duchas. No debe sorprendernos que, a partir de ese instante, los diálogos se presenten con mayor fluidez entre ellos, que sonrían y que se admiren entre sí una vez que ya se bañaron y que tienen la posibilidad de arreglarse y de mirarse al espejo. Tal vez recuerden lo que alguna vez vieron reflejado en el espejo o simplemente les guste lo que ven reflejado en él. Lo cierto es que, a pesar de que la comunicación entre la institución y las personas sin hogar es escasa, en este espacio se les brinda la posibilidad de dialogar y de comunicar los proyectos no ya como personas marginadas y asistidas, sino como sujetos ca-

El servicio de duchas, como se mencionó más arriba, es brindado por las organizaciones no gubernamentales y siempre se acompaña con el de ropería, por lo tanto, al momento de ducharse los sujetos también tienen la posibilidad de adquirir ropa acondicionada. Estos servicios solo están disponibles una vez por semana y los meses de verano permanecen cerrados.

10 En la institución donde se realizó el trabajo de campo se otorgaba la cantidad de quince turnos por mañana para la utilización de las duchas. "Si las duchas están habilitadas durante dos horas y son quince las personas que se tienen que bañar en solo dos duchas, significa que le corresponden dieciséis minutos a cada uno para llegar a tiempo. Esta simple ecuación también fue calculada por la parroquia y, en el redondeo, los dieciséis fueron reducidos a quince minutos 'reglamentarios' por persona" (Palleres 2004: 133). 
paces de percibir, de actuar y de proyectar en un contacto continuo con lo dialógico.

Para comprender la importancia que reviste el servicio de duchas en los sujetos, es conveniente señalar que para las instituciones y para vastos sectores de la sociedad, la persona sin hogar encarna la pasividad y la renunciación y, se supone que a través de estas, el individuo acepta su destino. Sin embargo, es necesario señalar que la historia de vida de los sujetos no se construye a través de biografías quebradas. Razón por la cual en los relatos siempre es posible marcar dos momentos mediados por una crisis: un antes (pasado) muy marcado y un ahora (presente) muy desdibujado. El pasado se encuentra caracterizado por el seno hogareño y todo lo que ello representa: la familia, la educación, las redes de contención, el trabajo, la estabilidad, la casa o el hogar. Por su parte, el presente aparece adverso e indeseable, se caracteriza por el ahora o la situación actual, por la inestabilidad y por la incertidumbre. De esta manera es difícil encontrar en los relatos la noción de un presente gozoso y -por lo general- estos expresan angustia, soledad, dolor y enfermedad. Cuando sus relatos se mantienen en el presente, estos son descriptivos y ejemplificativos de hechos o actividades. De tal modo, les es difícil hacer confluir el pasado y el presente, la actual situación los ha llevado a perder parte de su autoestima y los carga de impotencia. Sin embargo, en sus proyecciones hacia el futuro siempre rescatan y analizan vivencias del pasado que los condicionaron o determinaron a lo largo de la vida. No obstante, en el caso del servicio de duchas, temporalmente no hay pasado hacia un futuro, solo está el presente, es decir, se trata de un aquí y un ahora que les permite el reconocimiento de sí mismo. Aquí logran su pequeño espacio de reconocimiento que les es negado en los espacio públicos.

No es posible subestimar el hecho de que en el lugar elegido y al que retornan cada noche, siempre hay algo que se relaciona con las carencias y con las necesidades, no solo materiales, sino también afectivas. Pero al momento de realizar una revisión de su historia personal, en su mayoría, señalan que salvo por vivir en la calle no hay grandes cambios entre lo que ellos consideran que fue su vida normal y las circunstancias presentes, y esto responde a que, más allá de las condiciones materiales de la situación, ellos aseguran que conservan la moralidad y los valores aprendidos en su pasado. De hecho, la necesidad constante de ellos por marcar lo temporario de la actual situación -ya que el vivir en la calle solo es una mala jugada del destino que pronto será superada-, habla de la resistencia a ser rotuladas como personas de/en la calle. Por añadidura, continuamente buscan actividades que les permita sobrellevar la actual situación: la escritura de poemas, los dibujos y los relatos. Por medio de estas hallan una manera de expresar su sentir y una forma de reflexionar; reflejan su pasado a través de lo que perdieron, su presente habla de una situación nunca deseada y un futuro que se proyecta con temor a que la actual situación se repita. Si bien existe la conciencia de que el pasado es una etapa a la cual no se puede regresar, esto no significa que no sea tomada como anclaje para proyectarse hacia el futuro, el cual siempre es imaginado muy distinto al presente.

En otros términos, las personas sin hogar a través de actividades que les permitan la evasión, no solo encuentran aquel espacio de privacidad ansiado, sino que también se expresan sobrellevando alternativamente la situación actual. Así es como elaboran tácticas que son de resistencia y que les permiten resignificar su historia y, simultáneamente, crean y expresan la comprensión de uno sobre sí mismo.

\section{Resignificación del espacio y subjetividad}

El espacio urbano se transforma en un espacio vital en el transcurrir diario de las personas sin hogar, ya que en este se desarrollan las prácticas cotidianas y las interacciones entre los sujetos. Al llegar a este punto, se ha dado cuenta de cómo la movilidad por parte de las personas sin hogar nos habla de un proceso que atraviesa etapas a lo largo de la jornada, en las cuales es posible rastrear el trabajo, el hogar, el descanso y las relaciones interpersonales. El hecho de que este movimiento que refiere a un circuito cotidiano delimite rutinas muy marcadas a lo largo de una jornada, muestra que la vida en la calle no se caracteriza por el desorden y la renunciación, ya que no se trata de personas pasivas que ven 
como toda su trayectoria de vida se desploma a su alrededor. Por el contrario, estas transforman continuamente el espacio público no solo en y a través del mismo sino, principalmente, a través de su uso. Cada espacio utilizado y apropiado transitoriamente forma parte de un espacio más amplio, por este motivo es necesario comprender la movilidad y/o el movimiento constante a lo largo de la ciudad como parte de todo un proceso dinámico.

En el caso de las personas sin hogar, es posible definir el espacio público a través de dos formas de utilización. El primero de ellos se relaciona con el uso compartido, mientras que el segundo remite a la elección de un lugar determinado de la ciudad y a un uso personal del mismo. El conocimiento del espacio social es el que lleva a los sujetos a caminar a lo largo del día para llegar a destino, o bien para la búsqueda de un espacio que prometa un mínimo de privacidad o de tranquilidad. Por otra parte, el "estar aquí" del espacio individual, representa la totalidad de la actual situación. El concepto es interpretado no solo espacial sino también temporalmente, ya que remite directamente al presente y la satisfacción de necesidades. Es así que el "estar acá" y "ahora" es lo que imposibilita encontrarse bajo un techo cómodo y seguro, como el que en algún momento tuvieron en el seno de su hogar y de su familia, en el pasado. En el espacio individual es donde confluyen muchos sentimientos contradictorios. Sin embargo, es el lugar que les permite acceder a cierta privacidad, y si bien en este ellos son invadidos por sentimientos de temor y vergüenza, también encuentran evasión y vínculos interpersonales.

De esta manera, cargando de significación al uso de los espacios compartidos, es posible comprender la forma en que el espacio público es transformado y manipulado a través de continuas negociaciones llevadas a cabo por y entre las personas en un constante movimiento, a través de rutinas que le permiten moverse entre circuitos cotidianos y redes institucionales. Los sujetos en todo momento manipulan y negocian las distintas situaciones respondiendo no solo a su contexto social, sino, también, a su propia subjetividad. Poseen tácticas materiales (aseo diario, estructuras de cartón, obtención de comida y de ropa) y tácticas simbólicas que se materia- lizan a través de la memorización o escritura de poemas, de los dibujos y del relato en un continuo contacto con lo dialógico. Todas estas les permiten responder, manipular y transformar el espacio público a través de su uso. Las tácticas materiales están orientadas a resultados tangibles y observables, responden a la situación actual en tiempos y plazos inmediatos. Es decir, no son de resistencia, sino que son funcionales y pragmáticas. Si bien a través de la apropiación, del uso del espacio y de las tácticas materiales, las personas son fácil y visualmente identificables como personas en/de la calle, la necesidad constante por marcar la temporalidad de la actual situación, habla de la resistencia a través de tácticas simbólicas de ser rotuladas como tales. Las mismas son de resistencia y es el momento introspectivo que les permite ver con claridad su propia cotidianeidad, son una manera de responder a las estigmatizaciones pero también les permiten resignificar su pasado, su situación presente y su futuro. Conceptos como el amor y el respeto hacia sus padres, la felicidad de compartir momentos con su familia, la dignificación del trabajo y la importancia del hogar en la infancia, son traslados a una situación futura e incierta. Así la respuesta al "ser personas en la calle" se da a través de la moralidad y los valores inculcados en el seno hogareño conjugando, en menor medida, la experiencia adquirida con la vida en la calle. La forma en que ellos manifiestan y se posicionan en el hoy en continuas revisiones hacia el pasado rescatando valores y experiencias, les permite realizar una resignificación de lo que para ellos representa la noción espacial del estar aquí. No se construyen a sí mismos a través de un aquí y ahora, sino a través de un continuo movimiento en tiempos y lugares pasados cargados de seguridad, estabilidad y contención.

En este marco es donde el servicio de duchas adquiere mayor importancia, ya que aquí el presente se hace más patente y donde las personas sin hogar obtienen el reconocimiento y la privacidad que, en muchas ocasiones, no logran en los circuitos cotidianos. El acceso a un servicio que brindan las redes institucionales permite la confluencia y la conjugación del tiempo y del espacio social y del tiempo y el espacio individual, de lo universal (sociedad) y lo particular (sujeto) que 
ocurre una vez a la semana y solo por quince minutos.

\section{Consideraciones finales}

Los cambios en el rol del Estado a partir de la década de 1990, en la Argentina, sometió a vastos sectores de la población a mayores niveles de desigualdad y de exclusión social. En este contexto, el espacio urbano ha sufrido transformaciones profundas haciendo necesario el surgimiento de estudios renovados que ponderen no solo las variaciones en la composición social urbana, sino también la forma en que los distintos sectores de la población se conforman en el ámbito de las ciudades.

En el transcurso de este artículo se ha señalado que la utilización y la apropiación del espacio público por parte de las personas sin hogar se relacionan más con la posibilidad de crear vínculos y de acceder a las redes transitorias de asistencia que con su ubicación física propiamente dicha. Asimismo, el empleo de tácticas les permite no solo resistir y sobrellevar la situación actual, sino que también les brinda la posibilidad de superar el estigma y la victimización de sus personas. En este sentido, el espacio urbano no es una simple demarcación geográfica, sino que es entendido como poseedor de significado donde las relaciones sociales se producen y se reproducen. Si se defiende la idea de que el uso que se le da al espacio supera las conceptualizaciones de las fuerzas económicas y estructurales que lo conforman, entonces se comprenderá que el espacio público para las personas sin hogar no es solo el lugar donde pueden sobrevivir. Por el contrario, la utilización y apropiación del espacio público y la vida en la calle, moldean el transcurrir diario, la subjetividad y las formas de sociabilidad de las personas sin hogar. Los sujetos en todo momento resignifican tiempo y espacio individual, separan el aquí y el ahora de las orientaciones retrospectivas y prospectivas, ordenan y construyen el futuro sobre la base de experiencias pasadas por medio de la memoria y del proyecto.

No cabe duda que encontrarse con la necesidad de constituir su cotidianeidad enteramente en la calle produce un gran impacto en la subjetividad de las personas. La pérdida del hogar conlleva cambios profundos en la trayectoria biográfica de los sujetos y no solo deben realizar un gran esfuerzo para preservar un sentido de pertenencia y de autoestima, sino que también deben luchar continuamente contra los prejuicios y la discriminación. Las personas sin hogar son $-y$ cada vez más- posicionadas en situaciones inestables que les niega no solo el acceso a vivir en la ciudad de forma digna, sino también el derecho a la diferencia, a la elección de vínculos y a la intimidad. Es necesario superar las representaciones sociales que se han generado alrededor de las personas sin hogar, percibiéndolas como enfermas, peligrosas y culpables de su situación. Para ello será necesario desarrollar más estudios que den cuenta del proceso de construcción del espacio público en un contexto urbano, prestando especial atención al comportamiento en las calles, así como los múltiples y singulares actos de resistencia llevados a cabo por las personas sin hogar y su dedicación a confrontar y a transformar los lugares que ocupan y la capacidad de proyectar y de transformar de los sujetos.

\section{Bibliografía}

ALTHABE, G. (2005). "Final del juego 'la solidaridad': de ahora en más 'globalización, caridad y finanzas”. En HERNÁNDEZ, V.; HIDALGO, C. y STAGNARO, A. (comps). Etnografías Globalizadas. Sociedad Argentina de Antropología, Buenos Aires.

CALCAGNO, L. (1999). Los que duermen en la calle. Un abordaje de la indigencia extrema en la ciudad de Buenos Aires. Centro de documentación en políticas sociales 19 . Gobierno de la Ciudad de Buenos Aires, Buenos Aires.

HIDALGO DATTWYLER, R. (2007). “¿Se acabó el suelo en la gran ciudad? Las nuevas periferias metropolitanas de la vivienda social en Santiago de Chile. En Revista Eure, 98: 57-35, Santiago de Chile.

KAWASH, S. (1998). "The Homeless body". En Public Culture 10: 319-339.

MALANCA, P. (coord.) (2003). Personas sin techo. Algunas consideraciones psicológicas preliminares en el abordaje de trabajo de calle. Centro de Documentación en Políticas Sociales 28. Gobierno de la Ciudad de Buenos Aires, Buenos Aires. 
O'SULLIVAN, E. (2008). "Pathways through Homelessness: Theoretical Constructions and Policy Implications". En DOHERTY, J. y EDGAR, B. (ed.) In my Caravan, I feel like Superman. Essays in honour of Henk Meert 1963-2006. FEANTSA y Centre for Housing Research, Scotland.

PALlereS, G. (2004). Conjugando el Presente. Personas sin hogar en la Ciudad de Buenos Aires. Sociedad Argentina de Antropología, Buenos Aires.

PALLERES, G. (2009). "Parador Nocturno Retiro. Análisis del Programa de Paradores Nocturnos del Gobierno de la Ciudad de Buenos Aires para personas sin hogar". Tesina de Especialidad en Gestión de Proyectos Sociales. Universidad Nacional de Cuyo. Mendoza.

ROBERTS, B. (2007). "La estructura de la Pobreza”. En SARAVÍ, G. (ed.). De la pobreza a la exclusión social. Continuidades y rupturas de la cuestión social en América Latina. Prometeo, Buenos Aires.
SNOW, D. y ANDERSON, L. (1993). Down on their luck: A study of homeless street people. University of California Press, Berkeley.

SNOW, D. y MULCAHY, M. (2001). Space, Politics, and the Survival Strategies of the Homeless. En American Behavioral Scientist, 45: 49-169.

SVAMPA, M. (2005). La Sociedad excluyente. La Argentina bajo el signo del Neoliberalismo. Tauro, Buenos Aires.

VELÁSQUEZ, F. (ed.) (2007). Conversaciones sobre el derecho a la ciudad. Fedevivienda, Bogotá. 